\title{
Establishment of a protocol for the gene expression analysis of laser microdissected rat kidney samples with affymetrix genechips
}

\author{
Kerstin Stemmer ${ }^{\mathrm{a}, 1}$, Heidrun Ellinger-Ziegelbauer ${ }^{\mathrm{b}, 1}$, Kerstin Lotz $^{\mathrm{b}}$, \\ Hans-J. Ahr ${ }^{\mathrm{b}}$, Daniel R. Dietrich ${ }^{\mathrm{a}, *}$ \\ ${ }^{a}$ Environmental Toxicology, Faculty of Biology, University of Konstanz, PO Box-X918, Jacob-Burckhardtstrasse 25, D-78457 Konstanz, Germany \\ ${ }^{\mathrm{b}}$ Molecular and Special Toxicology, Bayer Healthcare AG, Germany
}

\begin{abstract}
Laser microdissection in conjunction with microarray technology allows selective isolation and analysis of specific cell populations, e.g., preneoplastic renal lesions. To date, only limited information is available on sample preparation and preservation techniques that result in both optimal histomorphological preservation of sections and high-quality RNA for microarray analysis. Furthermore, amplification of minute amounts of RNA from microdissected renal samples allowing analysis with genechips has only scantily been addressed to date. The objective of this study was therefore to establish a reliable and reproducible protocol for laser microdissection in conjunction with microarray technology using kidney tissue from Eker rats p.o. treated for 7 days and 6 months with 10 and $1 \mathrm{mg}$ Aristolochic acid/kg bw, respectively. Kidney tissues were preserved in RNAlater or snap frozen. Cryosections were cut and stained with either H\&E or cresyl violet for subsequent morphological and RNA quality assessment and laser microdissection. RNA quality was comparable in snap frozen and RNAlater-preserved samples, however, the histomorphological preservation of renal sections was much better following cryopreservation. Moreover, the different staining techniques in combination with sample processing time at room temperature can have an influence on RNA quality. Different RNA amplification protocols were shown to have an impact on gene expression profiles as demonstrated with Affymetrix Rat Genome 230_2.0 arrays. Considering all the parameters analyzed in this study, a protocol for RNA isolation from laser microdissected samples with subsequent Affymetrix chip hybridization was established that was also successfully applied to preneoplastic lesions laser microdissected from Aristolochic acid-treated rats.
\end{abstract}

Keywords: Laser capture microdissection; Laser microdissection and pressure catapulting; Kidney; RNA amplification; RNA integrity; Affymetrix; Microarray

\section{Introduction}

An exponentially increasing number of publications indicate that microarray technology is now an accepted and powerful tool for large-scale gene expression profiling. However, the outcome

Abbreviations: LCM, laser capture microdissection; LMPC, laser microdissection and pressure catapulting; RIN, RNA integrity number; IVT, in vitro transcription; AA, Aristolochic acid; PCA, Principle component analysis; p.o., per os.

* Corresponding author. Fax: +49 7531883170 .

E-mail addresses: kerstin.stemmer@uni-konstanz.de (K. Stemmer), heidrun.ellinger-ziegelbauer@bayerhealthcare.com (H. Ellinger-Ziegelbauer), kerstin.lotz@bayerhealthcare.com (K. Lotz),

hans-juergen.ahr@bayerhealthcare.com (H.-J. Ahr),

daniel.dietrich@uni-konstanz.de (D.R. Dietrich).

1 These authors contributed equally to this work. and interpretation of microarray studies can be strongly affected by inherent tissue heterogenicity. For example the kidney represents a tissue of high complexity with various structurally and functionally different compartments. It is therefore very difficult to causally link changes in gene expression patterns from whole kidney homogenates to cell-type-specific gene expression, the associated pathways and subsequent physiological effects. In addition, site-specific pathology-related gene expression changes, e.g., within a distinct tubular segment or a preneoplastic or neoplastic lesion can be diluted by the gene expression profile of other cell types in a whole tissue homogenate and thus not be readily detectable. Laser-assisted microdissection could be a useful tool to overcome such obstacles. Two of the most common technologies are laser capture microdissection (LCM) (Emmert-Buck et al., 1996) and laser microdissection and pressure catapulting (LMPC) (Schutze et 
al., 1998). Both technologies allow the dissection of cell populations, single cells or even sub-cellular structures from frozen or paraffin-embedded tissues. As the microdissected structures remain morphologically intact and macromolecules are not damaged by the laser beam, proteins, DNA or RNA can be isolated for subsequent analyses. However, the combination of laser-assisted microdissection and microarray technology has to cope with three main challenges: (1) minimizing RNA degradation over several tissue (section) processing steps, (2) preserving tissue morphology for accurate histological examination of lesions selected for microdissection and (3) maintaining the proportionality of specific RNA species after several rounds of RNA amplification, required to obtain sufficient RNA for chip hybridization.

It is important to conserve high-quality RNA, as degraded RNA can result in detection of an altered expression pattern (Spiess et al., 2003). RNA must therefore be protected during all processing steps, e.g., tissue harvesting, storage, sectioning, staining and fixation and finally during microdissection. Most of these steps are conducted at room temperature. The use of the commercially available stabilizing reagent RNAlater (e.g., from Ambion or Qiagen) could therefore be a promising tool for RNA preservation in cryo-fixed sections. As a first step of the experiments presented here, the use of RNAlater-preserved to snapfrozen tissue with respect to tissue morphology and RNA quality was compared. Furthermore, the influence of long term-storage, repeated use of same cryo-samples and different staining techniques on histomorphological tissue preservation and RNA quality was determined.

Besides maintaining tissue morphology and high-quality RNA, another challenge to be dealt with is the low amount of RNA obtained from microdissected material. Standard protocols for microarray analysis are based on starting amounts of $1-5 \mu \mathrm{g}$ total RNA. Yet laser-assisted microdissection yields at best nanograms of RNA. To overcome this obstacle, it is necessary to employ RNA amplification methods to generate the required microgram amounts of RNA. Linear one-cycle mRNA amplification by a T7-based in vitro transcription (IVT) protocol was first described by Van Gelder et al. (1990) and became the standard amplification and labeling protocol for the Affymetrix Chip technology. A second amplification round can be performed to further increase the RNA yield. However, a major concern remains as to whether the amplification via IVT maintains the proportionality of RNA species present in the original sample, since it was shown previously that gene expression ratios were not completely conserved between linearly amplified and non-amplified material (Baugh et al., 2001; Nygaard et al., 2005; Goley et al., 2004). Consequently, the comparability of one- and two-cycle amplification protocols on the gene expression profiles of rat kidney samples was elucidated and presented here. Kidney samples were taken from rats treated with vehicle control or Aristolochic acid (AA), a potent genotoxic renal toxicant and carcinogen that was previously shown to induce tumors in kidney, forestomach and urinary bladder of the rat (Mengs et al., 1982) as well as renal toxicity, progradient fibrosis and urothelial tumors in women exposed to AA in Chinese slimming teas (Vanherweghem et al., 1993, Nortier et al., 2000).
Based on the results of the experiments presented here, a protocol for RNA isolation from laser microdissected samples with subsequent Affymetrix chip hybridization was established that was also successfully applied to preneoplastic lesions laser microdissected from Aristolochic acid-treated rats.

\section{Methods}

Animal treatment and tissue preparation. Heterozygous Tsc2 mutant Eker rats were purchased at 6-8weeks of age from the University of Texas MD Anderson Cancer Center, Smithville, USA. Upon arrival, rats were allowed to acclimatize to laboratory conditions for 4 weeks. For the short-term experiment (7-day treatment) three randomly selected male Eker rats were gavaged daily with $10 \mathrm{mg}$ per $\mathrm{kg}$ BW Aristolochic acid (AA) per kg BW dissolved in $0.1 \mathrm{M}$ sodium bicarbonate, or with vehicle ( $0.1 \mathrm{M}$ sodium bicarbonate) alone. At the end of the experiment, rats were anesthetized and then killed via exsanguination subsequent to retrograde perfusion with PBS. Kidneys were collected, crosssectioned to 5-mm slices and either snap frozen with liquid nitrogen and stored at $-80{ }^{\circ} \mathrm{C}$ or fixed in RNAlater (Qiagen, Hilden, Germany) according to the manufacturer's instructions. For the latter procedure, kidney slices were incubated at room temperature for at least $1 \mathrm{~h}$, kept at $4{ }^{\circ} \mathrm{C}$ for up to 1 week, and then removed from the RNAlater reagent. From these kidney slices, kidney cortex was isolated and cortex and medulla stored separately in cryovials at $-80^{\circ} \mathrm{C}$. The RNA of the kidney cortex samples fixed in RNAlater was used for the comparison of the one- and two-cycle protocols shown in Figs. 3-5.

In the long-term exposure (6months) five male Eker rats per group were gavaged on 5 days per week for 6 months with $1 \mathrm{mg}$ per $\mathrm{kg}$ BW AA dissolved in $0.1 \mathrm{M}$ sodium bicarbonate and with vehicle ( $0.1 \mathrm{M}$ sodium bicarbonate) respectively. Following treatment, kidneys were collected as described for the short-term experiment (above) and then stored using two different protocols: one set of kidney cross-sections was stored in RNAlater as described above, and a second set of kidney slices was snap frozen in liquid nitrogen immediately after harvesting and subsequently stored at $-80{ }^{\circ} \mathrm{C}$. The kidney samples of these animals were used for results shown in (Figs. 1, 2, and 6) and in all tables

Sectioning and staining. Due to the high chaotrophic salt concentration of the RNAlater reagent, RNAlater preserved samples will not freeze in the cryostate and thus were thawed and then washed for $2 \times 5 \mathrm{~min}$ or $2 \times 15 \mathrm{~min}$ in ice-cold RNase-free PBS. For embedding samples were refrozen in the cryostate at $-20{ }^{\circ} \mathrm{C}$. To avoid interference of frozen tissue embedding reagent OCT (Cryoblock, medite Medizintechnik GmbH, Burgdorf, Germany) with laser efficiency and RNA isolation, samples were frozen only on a small drop of OCT instead of being fully embedded in the medium. Both RNAlater- and snap-frozen kidney samples were cut to $10-\mu \mathrm{m}$-thick sections on a cryotome. Sections were mounted onto RNase-free membrane-covered slides (PALM Microlaser GmbH, Bernried, Germany) and air-dried on ice for $20 \mathrm{~s}$. Subsequently, sections were fixed in ice-cold $70 \%$ ethanol (RNase-free) for $3 \mathrm{~min}$ and air-dried for 5 to $10 \mathrm{~min}$.

For H\&E (hematoxylin-eosin) staining, air-dried sections were stained with RNase-free Mayer's hematoxylin (Sigma Aldrich, Taufkirchen, Germany) for 3 min, rinsed with RNase-free tap water and counterstained with RNase-free eosin Y (Sigma Aldrich, Taufkirchen, Germany), for 3 min.

For cresyl violet staining, ethanol-fixed sections were stained with $1 \%(\mathrm{w} / \mathrm{v})$ cresyl violet acetate (Sigma Aldrich, Taufkirchen, Germany) in ACS-grade 100\% EtOH for $20 \mathrm{~s}$. Excess staining solution was removed by placing the sections on an absorbent surface.

Subsequent to H\&E or cresyl violet staining, sections were dehydrated by shortly dipping the sections in $70 \%$ and then $100 \%$ ethanol. For further information, see the customer protocol page of the PALM homepage at: www. palm-microlaser.com

Laser-assisted microdissection. For laser-assisted microdissection, a PALM laser microdissection and pressure catapulting (LMPC) system (PALM Microlaser $\mathrm{GmbH}$, Bernried, Germany) was used. Areas of variable size were dissected from H\&E-stained whole kidney sections (cresyl violet-stained sections were not used due to the poor histomorphological preservation of the sections, see below). The microdissected samples were collected in a non-contact and contamination-free manner, immediately lysed by addition of $300 \mu 1$ RLT 


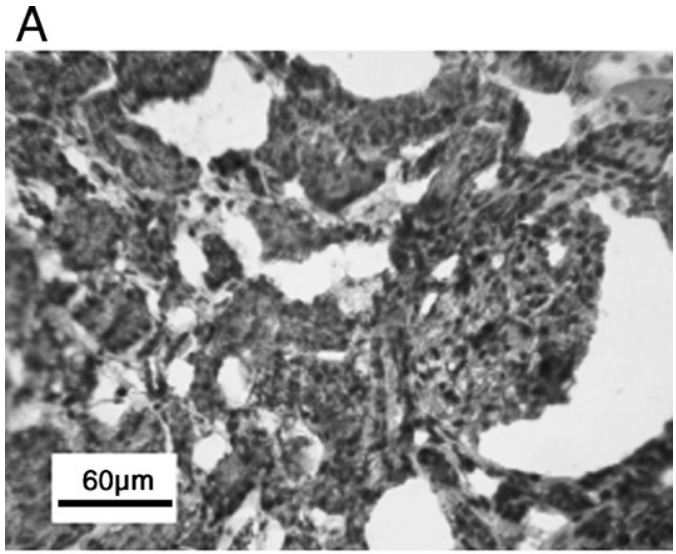

B

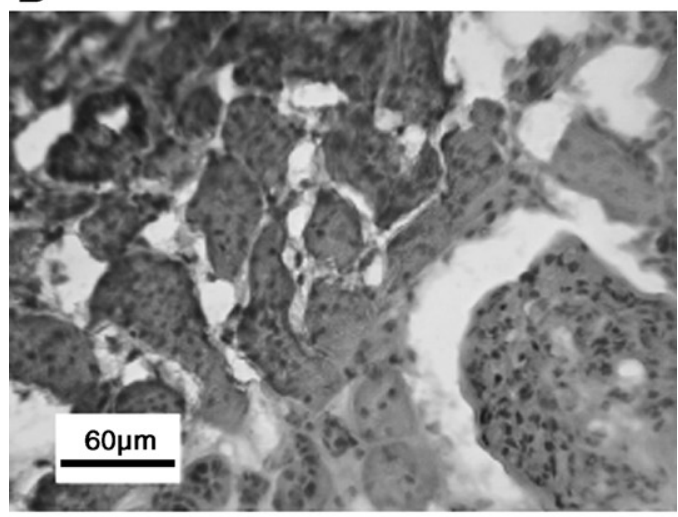

C

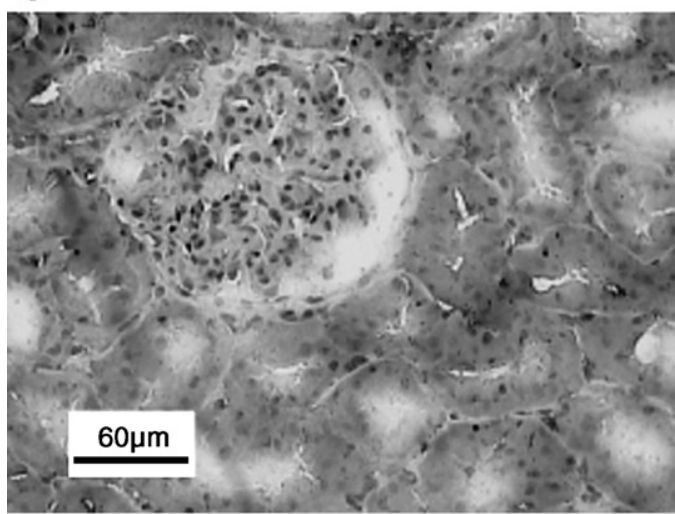

Fig. 1. Histology of H\&E-stained sections. (A) Section from RNAlater-fixed tissue after $2 \times 5$-min washes in PBS. (B) Section from RNAlater fixed tissue after $2 \times 15$-min washes in PBS. (C) Section from snap-frozen tissue, without an RNAlater presoak.

lysis buffer (RNeasy Micro Kit, Qiagen, Hilden, Germany) containing 1\% of $\beta$-mercaptoethanol (Sigma Aldrich, Taufkirchen, Germany) and stored at $-80^{\circ} \mathrm{C}$. For quality control, total RNA was isolated from additional, non-dissected and unstained whole sections from each kidney examined.

RNA isolation and quality control. RNA of microdissected tissue was isolated using the RNeasy Micro Kit (Qiagen, Hilden, Germany). RNA from whole kidney cortex immersed in RNAlater was isolated with Qiagen RNeasy Mini Kits. All isolations were performed according to the manufacturer's instructions. The quality of the total RNA was analyzed with the Agilent 2001 Bioanalyzer (Agilent Technologies GmbH, Germany) using RNA 6000 Nano or Pico chips. Agilent 2100 expert software was used to determine RNA quality, designated as RNA Integrity Number (RIN). RIN is calculated from the entire electrophoretic trace by Agilent 2100 expert software and thus represents the relative ratios of several RNA regions, e.g., the 5S rRNA region, the region between the 5S and $18 \mathrm{~S}$ rRNA and the $18 \mathrm{~S}$ and $28 \mathrm{~S}$ rRNA bands. Based on more than $1000 \mathrm{RNA}$ samples of different quality, an algorithm was generated that describes RNA integrity much more reliably then than the mere ratio of the ribosomal (18S and 28S rRNA) bands. A RIN of 7-10 is indicative of high RNA quality, whereas a RIN of 1 marks a completely degraded RNA sample (Schroeder et al., 2006).

For RNA concentrations $\geq 5 \mathrm{ng} / \mu \mathrm{l}$ (kidney cortex samples), the RNA quantity was determined using Ribogreen (Molecular Probes, Eugene, USA) For lower RNA concentrations (LMPC samples) the RNA quantity estimate by the Agilent 2100 expert software was utilized.

Data were statistically analyzed using GraphPad Prism 4 software with oneway ANOVA followed by Bonferroni's post test for multiple comparisons or Student's $t$-test for individual comparisons.

RNA amplification. For the synthesis of biotin-labeled cRNA with one or two rounds of amplification, the Affymetrix One-cycle and Two-cycle kits were used according to the manufacturer's instructions, the latter in conjunction with the Megascript T7 kit from Ambion (Austin, USA). $5 \mu \mathrm{g}$ of total RNA was used for the one-cycle protocol, during which the RNA is first reverse-transcribed in the presence of an oligo-dT primer coupled with a primer sequence for bacterial T7-polymerase. Following the synthesis of the second cDNA strand, this cDNA template is then transcribed with T7-polymerase in the presence of biotin-labeled ribonucleotides to yield biotin-labeled antisense cRNA. The concentration, corrected for input RNA, and the RNA quality was determined based on OD 260/280 measurement and the Agilent 2100 Bioanalyzer profile.

Up to $50 \mathrm{ng}$ of total RNA was employed as starting material with the twocycle protocol. After the first round of cDNA synthesis as described above, unlabeled antisense cRNA was synthesized with the Megascript T7 kit from Ambion (Austin, USA). The cRNA from this first round of amplification was quantified after column purification using Ribogreen. The first strand of the second round of cDNA synthesis was primed with random primers, whereas the second strand was primed with the oligo-dT-T7 primer as described above. Finally, biotin-labeled cRNA was synthesized, quantified and quality controlled as described for the one-cycle kit.

Affymetrix chip hybridization and expression profiling. $15 \mu \mathrm{g}$ of the fragmented biotin-labeled cRNA samples was hybridized on Affymetrix Rat Genome RAE230_2.0 Genechips according to the manufacturer's instructions.

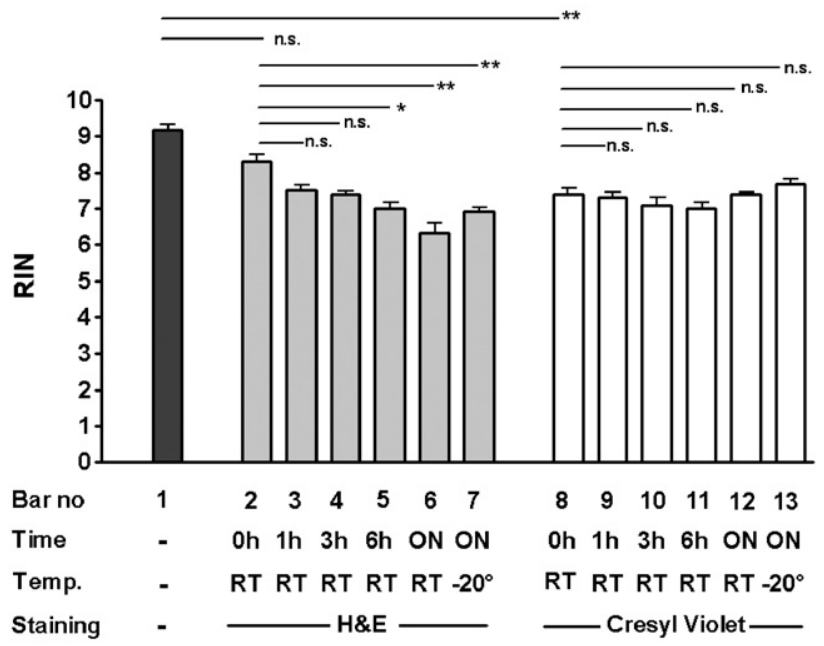

Fig. 2. Kidney tissue was frozen immediately after sacrifice and stored at $-80^{\circ} \mathrm{C}$ for 12 months. RNA was isolated from one complete kidney section of 3 independent replicate animals. Sections were stained with hematoxylin and eosin (H\&E, black bars) or cresyl violet (white bars) and subsequently incubated for $0-6 \mathrm{~h}$ or overnight $(\mathrm{ON})$ at $\mathrm{RT}$ or at $-20^{\circ} \mathrm{C}$. The control sections $(1 \mathrm{st}$ bar) were lysed immediately after sectioning. Significant differences, analyzed by Bonferroni's multiple comparison test, are indicated using significance levels of $p<0.05\left(^{*}\right)$ and $p<0.001(* *)$. 
RAE230_2.0 Genechips contain 31,000 probe sets represented by 11 pairs of 25 mer oligonucleotides. Each probe pair consists of a perfect match oligo (PM) complementary to the cRNA target sequence, and a mismatch oligo (MM) with a single base change in the middle to control for background and nonspecific hybridization. Raw data image files (DAT) were converted into CEL files by averaging the scan data from the 36 pixels per oligo set. With GeneData Expressionist Refiner software, dark and white spots, gradients and distortions were detected and corrected using the CEL file data. Using the MAS 5.0 statistical algorithms implemented in the Refiner software, the intensities of all 11 probe pairs were condensed to one intensity value per probe set associated with a statistical detection $p$-value calculated from the intensity differences of the PM and corresponding MM oligos. This $p$-value indicates how reliably a transcript is detected. After condensing, which also includes overall microarray background correction, the microarrays were scaled to an average signal intensity of 100 after excluding the highest $2 \%$ and lowest $2 \%$ of the data.

To select significantly deregulated genes between replicate sample groups representing kidney samples from short-term Aristolochic acid-treated and vehicle control Eker rats, a $t$-test $(p \leq 0.005)$ combined with a 1.7 -fold deregulation cut-off implemented in Genedata Expressionist Analyst software was employed. Genes with an intensity of expression $<15$ in all samples to be compared were excluded from the analysis. Principle component analysis (PCA) was performed to reduce the dimensionality of the expression data and to graphically display the major trends within different experimental set-ups in a three-dimensional space.

\section{Results}

\section{Tissue fixation and staining}

Influence of tissue preservation on histology of the sections and on RNA quality

Degradation of RNA can result in detection of altered gene expression patterns. Therefore, the question was asked whether tissue preservation with RNAlater would stabilize RNA in cryosections and whether the required PBS washing steps would negatively influence RNA stability. Two RNAlater fixed kidney pieces, each of three replicate animals was washed for $2 \times 5 \mathrm{~min}$ or $2 \times 15 \mathrm{~min}$ in RNase-free, ice-cold PBS. Subsequently, cryosections were cut and RNA was isolated from a complete unstained and an H\&E-stained section. As control, RNA was isolated from tissue homogenate of a third, unwashed RNAlaterfixed kidney piece. Table 1 depicts the RNA quality of the samples, displayed as RNA integrity number (RIN). The RNA quality is very good after $2 \times 5 \mathrm{~min}$ or $2 \times 15 \mathrm{~min}$ of washing in ice-cold PBS, with no significant difference to the control RNA from unwashed RNAlater-fixed tissue. The comparison with the RNA quality retrieved from snap-frozen tissue (Table 2, see below), RNAlater preservation provided for slightly lower RNA quality. Kidney morphology from RNAlater-fixed tissue sections was hardly discernible in unwashed kidney sections and also in sections washed for $2 \times 5 \mathrm{~min}$ (Fig. 1A). Further washing in PBS appeared to improve the preservation of renal tubular morphological characteristics of the renal cortical structure (Fig. 1B). However, cryosections from RNAlaterfixed tissue never achieved the histomorphological quality of cryosections from snap-frozen tissue even when stored up to 12 months at $-80{ }^{\circ} \mathrm{C}$ (Fig. 1C). Use of snap-frozen kidneys instead of RNAlater-fixed tissue appeared to allow a more precise histological and pathological examination, especially with regard to the identification of tubular structural detail, and thus to facilitate the structural identification required for LMPC.
Table 1

Influence of tissue preservation on RNA quality

\begin{tabular}{llll}
\hline Sample & Staining & Washing in PBS & RIN (mean and SD of 3 replicates) \\
\hline Control & N/A & N/A & $8.8 \pm 0.5$ \\
Section 1 & No & $2 \times 5 \mathrm{~min}$ & $8.8 \pm 0.6$ \\
Section 2 & H\&E & $2 \times 5 \mathrm{~min}$ & $8.0 \pm 0.1$ \\
Section 3 & No & $2 \times 15 \mathrm{~min}$ & $8.6 \pm 0.4$ \\
Section 4 & H\&E & $2 \times 15 \mathrm{~min}$ & $7.6 \pm 0.7$ \\
\hline
\end{tabular}

RNAlater-immersed kidney pieces from three replicate animals were washed for $2 \times 5 \mathrm{~min}$ or $2 \times 15 \mathrm{~min}$ in ice-cold PBS before freezing and cryosectioning. RNA was isolated from one unstained or one H\&E-stained section each. Control RNA was isolated directly from RNAlater-fixed, homogenized kidney cortex. RNA quality is displayed as mean RNA integrity number (RIN) \pm SD. One-way ANOVA and Bonferroni's post test $\left(p<0.05\left(^{*}\right)\right.$ and $\left.p<0.01\left({ }^{* *}\right)\right)$ suggested the absence of significant differences between the samples.

\section{RNA stability in snap-frozen kidney samples}

Since long-term storage of frozen tissue samples may have a negative impact on preservation of histological features and RNA stability, the influence of cryo-storage on histomorphological characteristics and RNA quality was investigated with snap-frozen tissue stored at $-80^{\circ} \mathrm{C}$ for 5 or 12 months post initial cryopreservation. RNA was isolated from renal cryosections of four independent animals. No significant decrease of RNA quality (RIN) or yield (ng) could be observed in frozen tissue stored up to 12 months at $-80{ }^{\circ} \mathrm{C}$ (Table 2). To elucidate whether repeated "thawing" of tissue samples from $-80{ }^{\circ} \mathrm{C}$ to cryostate temperature $\left(-18{ }^{\circ} \mathrm{C}\right.$ to $\left.-20{ }^{\circ} \mathrm{C}\right)$ has any influence on RNA stability or tissue morphology, RNA was isolated from complete cryosections of three replicate animals, that had been snap frozen, stored at $-80{ }^{\circ} \mathrm{C}$ for 12 months, and were then subjected to one or three cycles of "thawing" and refreezing. The mean RIN number of the respective samples only slightly decreased from $9.3 \pm 0.2$ to $8.5 \pm 0.2$ after the first and the third freeze-thaw cycle, respectively, yet still providing for high-quality RNA. Microscopic examination of multiple thawed samples demonstrated that tissue morphology was not adversely affected, thus also allowing pathological examination and histological differentiation (see also Fig. 1C, and above).

\section{Influence of the duration of section staining procedure on RNA quality}

Current working procedures require cryosections to be microdissected at room temperature. Depending on the number of areas that have to be collected from one specific cryosection, the actual dissection could last for several hours. However, both time and temperature could be critical factors for the isolation of high-quality RNA. RNA yield (ng) and quality (RIN) may in addition be adversely influenced by different staining methods. However, for kidney pathology H\&E staining is indispensable because it facilitates the distinction of morphological features as well as allows the detection of characteristic basophilic and eosinophilic preneoplastic and neoplastic lesions (Dietrich and Swenberg, 1991). Therefore, the influence of H\&E staining, temperature and LMPC duration on RNA yield and quality from H\&E-stained cryosections was investigated. In addition, RNA stability of H\&E-stained sections were compared with that of cresyl violet-stained sections, since cresyl violet is a recommended 
Table 2

Influence of long-term storage on RNA quality (RIN) and yield (ng RNA)

\begin{tabular}{lll}
\hline $\begin{array}{l}\text { Duration frozen at } \\
-80{ }^{\circ} \mathrm{C} \text { (months) }\end{array}$ & $\begin{array}{l}\text { RIN (mean } \pm \mathrm{SD} \\
\text { of } 4 \text { replicates) }\end{array}$ & $\begin{array}{l}\text { RNA ng yield (mean } \pm \mathrm{SD} \\
\text { of } 4 \text { replicates) }\end{array}$ \\
\hline 5 & $9.4 \pm 0.4$ & $681.6 \pm 103.3$ \\
12 & $9.2 \pm 0.2$ & $493.5 \pm 135.7$ \\
\hline
\end{tabular}

RNA was isolated from one complete section from the kidneys of 4 replicate animals. Kidney samples were snap-frozen rather than RNAlater immersed. The duration the tissues were kept frozen at $-80{ }^{\circ} \mathrm{C}$ as of initial freeze storing is shown in the first column.

staining method for subsequent RNA applications (customer protocol page at: http://www.palm-microlaser.com). The comparison of RNA immediately retrieved from unstained control sections and H\&E-stained sections demonstrated no significant influence of staining on RNA quality (Fig. 2, 1st and 2nd bar). Additional handling of the sections at room temperature, up to $3 \mathrm{~h}$, tendentially but not significantly decreased RNA quality. Additional handling time, $6 \mathrm{~h}$ to overnight, decreased RNA quality significantly with RIN number decreasing to values below 7.0 (Fig. 2, bar 2-6). In contrast cresyl violet staining resulted in a significant decrease of the RIN number directly after staining (Fig. 2, 1st and 8th bar). However, as of then RNA quality appeared to remain stable over time (Fig. 2, bar 8-12). Storing the differently stained sections overnight at $-20{ }^{\circ} \mathrm{C}$ in general appeared to reduce the rate of RNA degradation. When comparing the RIN numbers of the H\&E with the cresyl violetstained sections within the first $3 \mathrm{~h}$ of handling at room temperature, thus representing a reasonable processing time, $\mathrm{H} \& \mathrm{E}$ sections provided for a higher RNA quality than the corresponding cresyl violet-stained sections. Therefore, RNA of high quality that allows additional RNA processing, e.g., chip hybridization, can be best obtained from H\&E-stained cryosections within $3 \mathrm{~h}$ post staining.

\section{RNA quality and yield from pooled microdissected samples}

To investigate whether the area of pooled microdissected samples had an influence on RNA quality and yield, areas of $1 \mathrm{~mm}^{2}$ and $2 \mathrm{~mm}^{2}$ were laser microdissected from H\&E-stained cryosections and analyzed. These analyses as shown in Table 3 suggested that total RNA from pooled sections were of comparably high quality (RIN >8.0). RNA yields from both $1 \mathrm{~mm}^{2}$ or $2 \mathrm{~mm}^{2}$ sections (Table 3 , also see below) indicated that microdissection of an approximate area of at least $2 \mathrm{~mm}^{2}$ was necessary to achieve the minimum amount of RNA (5ng to $100 \mathrm{ng}$ of total RNA) required for the two-cycle amplification

Table 3

RNA quality and yields from pooled microdissected samples

\begin{tabular}{lll}
\hline $\begin{array}{l}\text { Pooled area } \\
\left(\mathrm{mm}^{2}\right)\end{array}$ & $\begin{array}{l}\text { RIN } \\
(\text { mean } \pm \text { SD of 3 replicates })\end{array}$ & $\begin{array}{l}\text { Yield ng RNA } \\
(\text { mean } \pm \text { SD of 3 replicates })\end{array}$ \\
\hline 1 & $8.0 \pm 0.2$ & $3.4 \pm 0.3$ \\
2 & $8.3 \pm 0.4$ & $8.8 \pm 1.7$ \\
\hline
\end{tabular}

RNA was isolated from pooled microdissected samples of an overall area of $1 \mathrm{~mm}^{2}$ or $2 \mathrm{~mm}^{2}$ protocol. For area comparison, a cross-sectioned glomerulus has an average area of $0.02 \mathrm{~mm}^{2}$.

\section{RNA amplification and microarray hybridization}

\section{Influence of one-cycle and two-cycle amplification on the expression profile}

Laser-assisted microdissection only yielded nanograms of RNA, thus necessitating global mRNA amplification for an intended subsequent microarray analysis. Therefore, microarray experiments with different starting amounts of kidney RNA were subjected to one- or two-cycle amplification to investigate the influence of one-cycle or two-cycle amplification on the expression pattern. Fig. 3 depicts that the RNA amplification factor is highly dependent on the starting amount of RNA. Using $10 \mathrm{ng}$ of starting RNA, two-cycle amplification led to a 12,000- to 14,000 -fold increase in RNA yield. With $50 \mathrm{ng}$ of starting RNA, the same protocol resulted in 2500- to 3000-fold RNA yield. One-cycle amplification with $5 \mu \mathrm{g}$ of starting RNA led to a 20 - to 30 -fold increase of the RNA yield. Irrespective of the amount of starting RNA or the amplification protocol employed, the RNA yield was comparable (Fig. 3). The latter observation was tested with a Bonferroni's multiple comparison test which suggested that no significant differences can be observed. This further suggests a saturation of the amplification process, an artifact that could lead to preferential amplification of certain genes and consequently in the generation of aberrant expression profiles. To test this hypothesis, the expression profiles of above samples were analyzed using Affymetrix Rat Genome RAE230_2.0 arrays. The heat maps in Fig. 4 depict the ratios of gene expression calculated by dividing the expression values in the single AA-treated replicate samples by the mean expression value of the corresponding time-matched control replicates. Most of the genes selected with both the one-cycle protocol and the twocycle protocol starting with $10 \mathrm{ng}$ were similarly upregulated after one- and two-cycle amplification. Only ca. $20 \%$ of the genes were qualitatively differently expressed in the two protocols, i.e., approximately $80 \%$ of the genes were found to be significantly and similarly deregulated following a one-cycle and two-cycle amplification. Furthermore, a preliminary analysis of specific genes that were deregulated following AA

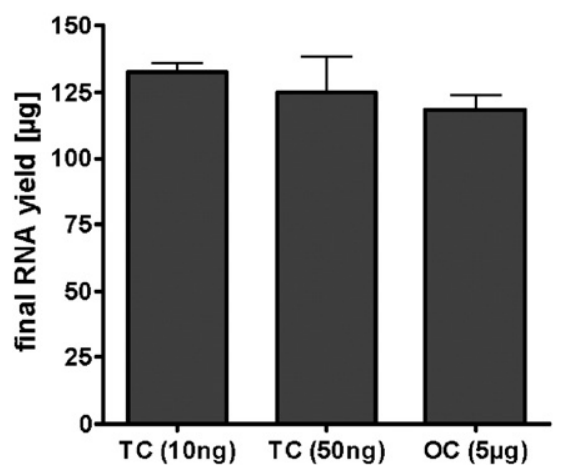

Fig. 3. Comparison of total RNA yields after either one-cycle (OC) amplification with $5 \mu \mathrm{g}$ of starting RNA or two-cycle (TC) amplifications with $10 \mathrm{ng}$ or $50 \mathrm{ng}$ of starting material, respectively. Data represent mean yield $\pm \mathrm{SD}$ ( $n=6$ replicates). 

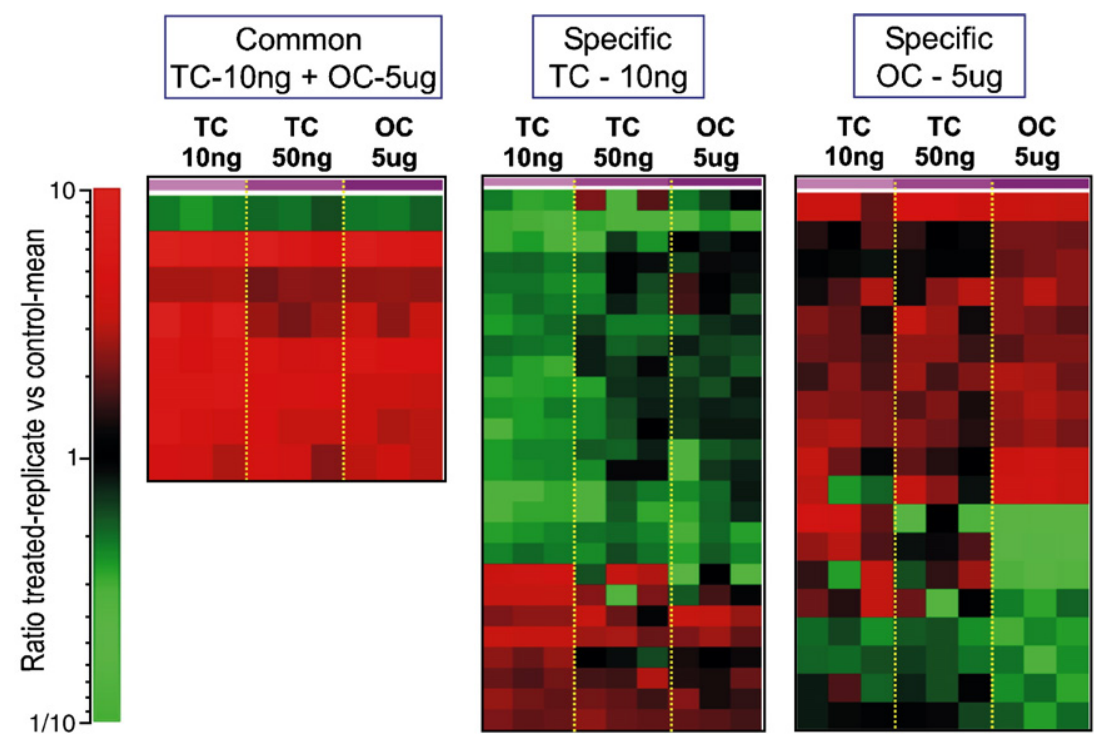

Fig. 4. Expression profiles of differentially expressed genes. Genes that were assigned to be differentially expressed after one (OC)- or two (TC)-cycle amplification of the indicated amount of RNA from rat kidney after treatment with Aristolochic acid for 7 days were subjected to one-dimensional cluster analysis using Euclidian as distance metric. The heat maps show ratios of gene expression calculated by dividing the expression values in the single treated replicate samples through the mean expression value of all corresponding time-matched control replicates. The left diagram depicts the genes commonly selected with the TC (10ng) and the OC protocol, the diagram in the middle those specifically selected with the TC $(10 \mathrm{ng})$ protocol, and the diagram on the right those specifically selected with the OC protocol. The red color illustrates upregulated and green downregulated genes always in comparison to the corresponding control, as indicated by the color scale.

treatment demonstrated that the same main biological pathways were identified in the two amplification protocols thus suggesting that no preferential/aberrant gene expression is observed due to differing amplification protocols (Stemmer et al., in preparation).

\section{Reproducibility of RNA amplification and microarray hybridization}

Absolute expression profiles of all genes on a microarray may be influenced by the reagent lots used. Therefore, hybridization of cRNA prepared at different dates with different reagent lots was carried out at varying dates. This was done with RNA from the short-term AA-treated and vehicle control samples described above and whose separation in the three-dimensional space (PCA) is illustrated in Fig. 5. The principle components after the one- and two-cycle protocols separate from each other only in one dimension, whereas the principle components of treated and control samples separate in another dimensions namely the amplification procedure dimension and the treatment dimension. The $20 \%$ of the total deregulated genes observed that differed between the two amplification protocols may have been responsible for the shift in the dimension observed in the PCA (Fig. 5A). From these observations it can be concluded that genes deregulated between treated and control samples can be detected reliably, as reported above, but that microarrays from treated samples must be compared with control microarrays employing the identical amplification and hybridization protocol. Preparation of cRNA at different dates using separate reagent kits leads to a separation of the overall expression profile in PCA space (Fig. 5B). This appears mainly due to the separate
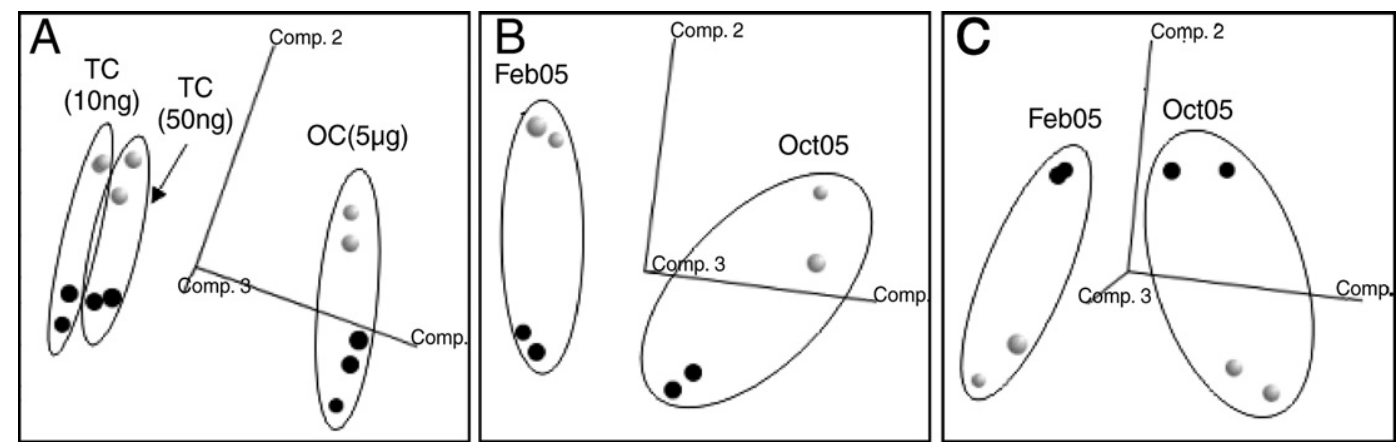

Fig. 5. Principal components analysis based on the expression of all genes of the RAE230A subset (15923 probe sets) on the RAE230_2.0 chip. Black and grey dots depict kidney samples of control and of Aristolochic acid-treated rats, respectively. Ellipses indicate samples processed with identical protocols. (A) Different amounts of the same RNA sample subjected to one-cycle (OC) or two-cycle (TC) amplification. (B) 10ng of the same RNA sample subjected to TC amplification and chip hybridization at two different dates (Feb05, Oct05). (C) 10ng of the same RNA sample subjected to TC amplification at two different dates (Feb05, Oct05), yet hybridized on the same day. 
cRNA synthesis and not as a result of microarray hybridization at different dates, an interpretation that is corroborated by the PCA in Fig. 5C, for which two separate cRNA preparations were hybridized on the same day, yielding a very similar dimensional separation as observed for the PCA in Fig. 5B. Despite the influence of amplification protocols and possibly hybridization, profile separation between treated and control is always maintained. The conclusion of the above findings thus would be that in order to select differentially expressed genes due to a given treatment, samples of the control and treatment groups must be prepared using the identical protocol and within the same protocol handling run.

\section{Performance of the established protocol}

To test the reliability of above protocol RNA was isolated from microdissected samples of control and AA exposed male Eker rats $(n=3)$ of the 6-month experiment for Affymetrix chip hybridization. Three different types of renal lesions, namely basophilic atypical tubuli (bAT) basophilic hyperplasia (bHA) and chronic progressive nephropathy (CPN), as well as healthy tissue (HT) of the same rats were excised via LMPC. RNA ( $5 \mathrm{ng}$ ) was isolated from each sample type (lesion type or HT) and analyzed on Affymetrix RAE230_2.0 chips (one chip per lesion type or HT and animal). Amplification factors were comparable for excised sample types. A boxplot, displaying the absolute expression values after scaling to a mean target expression value of 100 per microarray, was used as a quality check of the hybridized chips. No outliers or significant variations in chip hybridizations were found. The data thus demonstrated that the procedure developed generated reproducible and reliable gene expression determinations.

\section{Discussion}

Gradual degradation of RNA can introduce a variable bias in the quantification and amplification of gene transcripts. This degradation, especially during a protocol encompassing various processing steps, can severely affect the results of downstream applications, e.g., gene expression profiling. It is therefore crucial to protect the RNA from degradation in order to obtain reliable gene expression results. RNA integrity numbers can be used as standard to define an individual minimum threshold of RNA quality. For the study presented here a RIN number threshold of 7.0 was used. This threshold was chosen based on recommendations by the manufacturer Agilent, who also provides the RIN-calculating software (Schroeder et al., 2006). The comparison of different preservation and staining techniques demonstrated that RNA with the required high quality as well as optimal conservation of tissue morphology can be obtained from cryosections of snap-frozen tissue samples that were stained with H\&E. The latter findings, however, stand in contrast to previously published data by Ellis et al. (2002) where RNAlater-fixed and PBS-washed pancreas samples provided reasonably good tissue morphology. Thus caution is advised as to generalizations of fixation and processing protocols, while emphasizing that protocol optimization procedures, as presented here, are advisable for every animal and organ prior to em- barking on expression profile analyses of microdissected tissue samples.

From all parameters tested, processing of cryosections at room temperature had the most adverse effect on RNA quality, emphasizing that in order to conserve high-quality RNA from stained cryosections, $3 \mathrm{~h}$ of processing should not be exceeded.

In addition to maintenance of RNA quality, standardization of RNA amplification and chip hybridization is of utmost importance. Indeed, optimization of amplification protocols also entailed starting with comparable RNA quantities in order to avoid saturation effects, as demonstrated here. Due to the tubular structure of the kidney, microdissected areas of comparable size

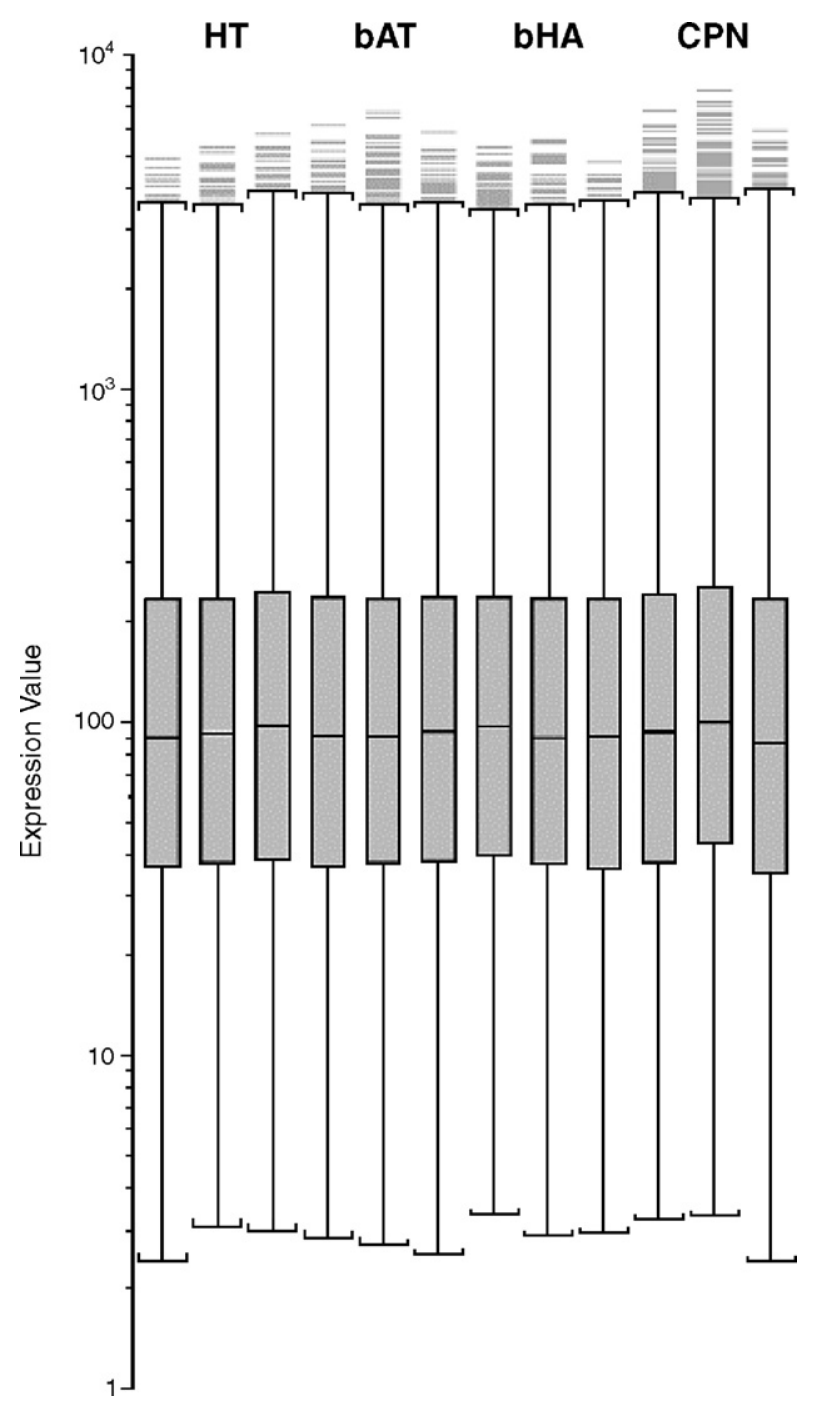

Fig. 6. Boxplot analysis of Affymetrix genechip data obtained from microdissected renal samples. $5 \mathrm{ng}$ kidney RNA isolated from various sample types of rats $(n=3)$ treated with AA for 6 months. Sample types: healthy tissue (HT), basophilic atypical tubuli (bAT), basophilic hyperplasia (bHA), and chronic progressive nephropathy (CPN). Isolated RNA was subjected to twocycle amplification and Affymetrix RAE230_2.0 chip hybridization. After scaling to a target intensity of 100 , the expression intensity distribution in each Affymetrix chip, corresponding to a given sample type and animal, was visualized in a boxplot format. The box itself is limited by lower and upper hinges, which correspond to the $25 \%$ and $75 \%$ quantiles, respectively. 
$\left(\mathrm{mm}^{2}\right)$ can vary in RNA yields. A minimum area of $2 \mathrm{~mm}^{2}$ should be microdissected for reliable RNA quantification and subsequent two-cycle amplification. In addition, it was demonstrated that samples should be prepared with an identical protocol during the same time frame and quality analysis by box plot analysis, to ensure reliable gene expression data. In conclusion, a protocol for laser-assisted microdissection in conjunction with Affymetrix microarray technology was established that allows investigation of rat kidney gene expression profiles with high reproducibility and reliability (Fig. 6). This protocol is described in detail in Appendix A.

\section{Acknowledgments}

We would like to thank the Federal Ministry of Education and Research (BMBF: 0313024) for funding the project, $\mathrm{T}$. Lampertsdoerfer and G. von Scheven for their skillful assistance during the whole animal experiment, E. O'Brien and A.H. Heussner for help with the animal sacrifice.

\section{Appendix A. List 1: Recommended protocol for laser-assisted microdissection in conjunction with Affymetrix microarray technology}

1. Collect kidneys from sacrificed animals and section each kidney into ca. $5 \mathrm{~mm}$ replicate slices.

2. Wrap kidney slices into sterile (oven baked-RNAse free) aluminum foil and immediately snap-freeze samples in liquid nitrogen.

3. Use samples directly or store at $-80^{\circ} \mathrm{C}$.

4. For preparation of cryosections, cool cryostate to $-20^{\circ} \mathrm{C}$ and "thaw" the respective sample from $-80{ }^{\circ} \mathrm{C}$ to cryostate temperature. It is possible to refreeze the samples at $-80{ }^{\circ} \mathrm{C}$ for later use, however 3 "freeze and thaw" cycles should not be exceeded.

5. Freeze samples onto a small drop of OCT embedding media in the cryostate.

6. Prepare $10-\mu \mathrm{m}$ cryosections and mount onto special RNase-free membrane covered slides, recommended for the respective microdissection system, e.g., the LMPC system from PALM Microlaser GmbH, Bernried Germany.

7. Air dry freshly cut sections for $20 \mathrm{~s}$ in the cryostate and subsequently fix them in $-20{ }^{\circ} \mathrm{C}$ cold ethanol $(70 \%$ in RNase-free $\mathrm{ddH}_{2} \mathrm{O}$ ) for $3 \mathrm{~min}$.

8. Air dry sections for $10 \mathrm{~min}$ at RT.

9. Stain sections with RNase-free, ice-cold Mayer's hematoxylin for $3 \mathrm{~min}$.

10. Rinse with RNase-free tap water for $3 \mathrm{~min}$.

11. Counterstain with RNase-free, ice-cold eosin Y for $3 \mathrm{~min}$.

12. Remove excess staining solution on absorbent surface and dehydrate section by shortly dipping into ice-cold $70 \%$ and $100 \%$ ethanol.

13. Air-dry sections at RT and directly use for laser-assisted microdissection employing, e.g., the PALM LMPC system (PALM Microlaser GmbH, Bernried Germany).

14. For RNA isolation from microdissected kidney samples pool a minimum area of $2 \mathrm{~mm}^{2}$ and collect samples into an appropriate sampling cup of the respective microdissection system.

15. After completion of the sample collection, immediately lyse pooled samples via addition of $300 \mu 1$ lysis buffer containing 1\% $\beta$-mercaptoethanol (e.g., component of the RNeasy Micro Kit, Qiagen, Hilden, Germany).

16. Do not exceed $3 \mathrm{~h}$ between staining and lysis of pooled samples.

17. Isolate RNA using e.g. RNeasy Micro Kit (Qiagen, Hilden, Germany) according to the manufacturer's instruction.

18. Analyze total RNA quality and estimate quantity, using an Agilent Bioanalyzer. For later amplification and hybridization procedures, use only RNA samples with a RIN numbers $>7.0$.

19. Amplify RNA and convert to biotin-labeled cRNA using e.g. the Affymetrix Two-cycle Target labeling kit according to the manufacturers instructions. Always use same starting quantity of RNA, e.g., $5 \mathrm{ng}$ or $10 \mathrm{ng}$ RNA.

20. Use $15 \mu \mathrm{g}$ biotin-labeled cRNA samples for Affymetrix chip hybridization and process the hybridizations according to the manufacturer's instruction.

\section{References}

Baugh, L.R., Hill, A.A., Brown, E.L., Hunter, C.P., 2001. Quantitative analysis of mRNA amplification by in vitro transcription. Nucleic Acids Res. 29, E29.

Dietrich, D.R., Swenberg, J.A., 1991. Preneoplastic lesions in rodent kidney induced spontaneously or by non-genotoxic agents: predictive nature and comparison to lesions induced by genotoxic carcinogens. Mutat. Res. 248, 239-260.

Ellis, M., Davis, N., Coop, A., Liu, M., Schumaker, L., Lee, R.Y., Srikanchana, R., Russell, C.G., Singh, B., Miller, W.R., Stearns, V., Pennanen, M., Tsangaris, T., Gallagher, A., Liu, A., Zwart, A., Hayes, D.F., Lippman, M.E., Wang, Y., Clarke, R., 2002. Development and validation of a method for using breast core needle biopsies for gene expression microarray analyses. Clin. Cancer Res. 8, 1155-1166.

Emmert-Buck, M.R., Bonner, R.F., Smith, P.D., Chuaqui, R.F., Zhuang, Z., Goldstein, S.R., Weiss, R.A., Liotta, L.A., 1996. Laser capture microdissection. Science 274, 998-1001.

Goley, E.M., Anderson, S.J., Menard, C., Chuang, E., Lu, X., Tofilon, P.J., Camphausen, K., 2004. Microarray analysis in clinical oncology: preclinical optimization using needle core biopsies from xenograft tumors. BMC Cancer 4, 20

Mengs, U., Lang, W., Poch, J.-A., 1982. The carcinogenic action of Aristolochic acid in rats. Arch. Toxicol. 51, 107-119.

Nortier, J.L., Martinez, M.C., Schmeiser, H.H., Arlt, V.M., Bieler, C.A., Petein, M., Depierreux, M.F., De Pauw, L., Abramowicz, D., Vereerstraeten, P., Vanherweghem, J.L., 2000. Urothelial carcinoma associated with the use of a Chinese herb (Aristolochia fangchi). N. Engl. J. Med. 342 (23), 1686-1692.

Nygaard, V., Holden, M., Loland, A., Langaas, M., Myklebost, O., Hovig, E., 2005. Limitations of mRNA amplification from small-size cell samples. BMC Genomics 6, 147.

Schroeder, A., Mueller, O., Stocker, S., Salowsky, R., Leiber, M., Gassmann, M., Lightfoot, S., Menzel, W., Granzow, M., Ragg, T., 2006. The RIN: an RNA integrity number for assigning integrity values to RNA measurements. BMC Mol. Biol. 7, 3.

Schutze, K., Posl, H., Lahr, G., 1998. Laser micromanipulation systems as universal tools in cellular and molecular biology and in medicine. Cell. Mol. Biol. (Noisy-le-grand) 44, 735-746.

Spiess, A.N., Mueller, N., Ivell, R., 2003. Amplified RNA degradation in T7- 
amplification methods results in biased microarray hybridizations. BMC Genomics 4, 44.

Van Gelder, R.N., von Zastrow, M.E., Yool, A., Dement, W.C., Barchas, J.D., Eberwine, J.H., 1990. Amplified RNA synthesized from limited quantities of heterogeneous cDNA. Proc. Natl. Acad. Sci. U. S. A. 87, 1663-1667.
Vanherweghem, J.L., Depierreux, M., Tielemans, C., Abramowicz, D., Dratwa, M., Jadoul, M., Richard, C., Vandervelde, D., Verbeelen, D., VanhaelenFastre, R., et al., 1993. Rapidly progressive interstitial renal fibrosis in young women: association with slimming regimen including Chinese herbs. Lancet 341 (8842), 387-391. 